\title{
The prognostic value of preoperative serum CA724 for CEA- normal colorectal cancer patients
}

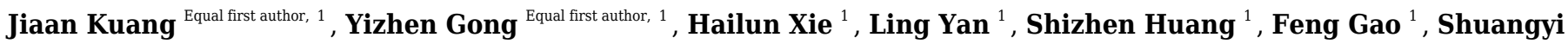 \\ Tang $^{2}$, Jialiang Gan ${ }^{\text {Corresp. } 1}$ \\ ${ }^{1}$ Department of Colorectal Anal Surgery, The First Affiliated Hospital of Guangxi Medical University, Nanning, Guangxi, China \\ 2 Department of Pharmacy, The First Affiliated Hospital of Guangxi Medical University, Nanning, Guangxi, China \\ Corresponding Author: Jialiang Gan \\ Email address: gj15172@163.com
}

Background and Purpose: There had been no recognized serum tumor marker to predict the prognosis of colorectal cancer (CRC) patients with normal preoperative serum carcinoembryonic antigen (CEA) levels. The purpose of this study was to determine whether preoperative serum carbohydrate antigen 724 (CA724) was of predictive function for the prognosis of CRC patients with normal CEA levels.

Methods: The medical records of 295 CRC patients with normal CEA levels who underwent surgery at the Department of Colorectal Anal Surgery of the First Affiliated Hospital of Guangxi Medical University (Guangxi, China) between September 2012 and September 2014 were retrospectively reviewed. Chisquare test was used to test the correlation between preoperative serum CA724 levels and clinical features. Kaplan-Meier curves were conducted to calculate the overall survival (OS) rate and disease-free survival (DFS) of patients. Cox regression analysis was applied to conduct univariate and multivariate analysis of the following four preoperative serum tumor makers namely CA724, carbohydrate antigen 199 (CA199), carcinoembryonic antigen 125 (CA125), carcinoembryonic antigen 242 (CA242) and clinical features. Nomograms for prognostic parameter of OS and DFS were developed using R v3.2.5.

Results: In the Chi-square test, only pathological node stage ( $\mathrm{pN}$ stage) $\left(X^{2}=14.514, P=0.001\right)$ and differentiation ( $X^{2}=10.712, P=0.001$ ) were associated with serum CA724 levels. In the Kaplan-Meier analysis, the results revealed that the OS and DFS in patients with high CA724 was poorer than those with normal. In the multivariate Cox regression analysis of OS and DFS, only pT stage, pN stage, metastasis and serum CA724 were independent prognostic risk factors for CRC patients with normal CEA levels.

Conclusion: Preoperative serum CA724 might serve as a potential prognostic factor for CRC patients with normal serum CEA levels. 
1 The prognostic value of preoperative serum CA724 for CEA-normal colorectal cancer 2 patients

3 Jiaan Kuang $^{1 *}$, Yizhen Gong ${ }^{1 *}$, Hailun $\mathrm{Xie}^{1}$, Ling Yan ${ }^{1}$, Shizhen Huang ${ }^{1}$, Feng Gao ${ }^{1}$, Shuangyi

$4 \quad$ Tang $^{2}$, Jialiang Gan ${ }^{1 \#}$

$5 \quad *$ Equal contributors

6

$7{ }^{1}$ Department of Colorectal Anal Surgery, The First Affiliated Hospital of Guangxi Medical

8 University, Nanning, Guangxi, China.

92 Department of Pharmacy, The First Affiliated Hospital of Guangxi Medical University,

10 Nanning, Guangxi, China.

\#Corresponding Author: Prof. Jialiang Gan, Department of Colorectal Anal Surgery, The First

13 Affiliated Hospital of Guangxi Medical University, 6 Shuangyong Road, Nanning 530021,

14 Guangxi, China. Tel: (+86)-13878155172. E-mail: ganjialiang@gxmu.edu.cn.

16 Keywords: CA724, normal CEA levels, colorectal cancer, prognosis

17 


\section{Abstract}

24 Background and Purpose: There had been no recognized serum tumor marker to predict the prognosis of colorectal cancer (CRC) patients with normal preoperative serum carcinoembryonic antigen (CEA) levels. The purpose of this study was to determine whether preoperative serum carbohydrate antigen 724 (CA724) was of predictive function for the prognosis of CRC patients with normal CEA levels.

Methods: The medical records of $295 \mathrm{CRC}$ patients with normal CEA levels who underwent surgery at the Department of Colorectal Anal Surgery of the First Affiliated Hospital of Guangxi Medical University (Guangxi, China) between September 2012 and September 2014 were retrospectively reviewed. Chi-square test was used to test the correlation between preoperative serum CA724 levels and clinical features. Kaplan-Meier curves were conducted to calculate the overall survival (OS) rate and disease-free survival (DFS) of patients. Cox regression analysis was applied to conduct univariate and multivariate analysis of the following four preoperative serum tumor makers namely CA724, carbohydrate antigen 199 (CA199), carcinoembryonic antigen 125

37 (CA125), carcinoembryonic antigen 242 (CA242) and clinical features. Nomograms for 
38

39 Results: In the Chi-square test, only pathological node stage ( $\mathrm{pN}$ stage) $\left(\mathrm{X}^{2}=14.514, P=0.001\right)$

prognostic parameter of OS and DFS were developed using R v3.2.5.

40 and differentiation $\left(\mathrm{X}^{2}=10.712, P=0.001\right)$ were associated with serum CA724 levels. In the

41 Kaplan-Meier analysis, the results revealed that the OS and DFS in patients with high CA724 was

42 poorer than those with normal. In the multivariate Cox regression analysis of OS and DFS, only

43 pT stage, $\mathrm{pN}$ stage, metastasis and serum CA724 were independent prognostic risk factors for CRC

44 patients with normal CEA levels.

45 Conclusion: Preoperative serum CA724 might serve as a potential prognostic factor for CRC 46 patients with normal serum CEA levels.

\section{Introduction}

Colorectal cancer (CRC) is the third most common malignancy and the second most common cause of cancer-related mortality worldwide(Bray et al. 2018). In China, CRC is the fifth most common malignancy, and the incidence of this disease continues to increase gradually(Chen et al.

51 2016a). Surgical resection is still a mainstay of curative treatment for CRC(Benson et al. 2018). However, survival after radical resection in some patients was poor because of recurrence and metastasis. Some markers to predict prognosis had been discovered for screening of risk for recurrence or metastasis(Feng et al. 2018; Nakamura \& Yoshino 2018). But their performance on clinic appliance was imperfect and some testing was only implemented in more comprehensive medical institutions due to the technology and expense. Pathologic stage is an important prognostic 
57 factor, but its adequacy had been recently questioned(Lea et al. 2014; Li et al. 2014). Therefore, it

58 was essential to identify other markers to predict the prognosis of CRC patients.

59 Serum tumor markers are produced and released during tumorigenesis, and the levels of serum

60 tumor markers may be indicative of tumor growth(Jo et al. 2013). Many valuable studies had

61 suggested that preoperative serum carcinoembryonic antigen (CEA) levels could be used as

62 independent risk factors to estimate the prognosis of CRC patients(Chen et al. 2017; Kozman et

63 al. 2018; Peng et al. 2015). However, the high rate of serum CEA in CRC patients was less than

$6450 \%$ in some clinical trials(Huang et al. 2018; Huang et al. 2017; Shinkins et al. 2017).

65 Furthermore, at present, there was no recognized reliable serum tumor marker for prognostic use

66 in serum CEA-normal CRC patients. Carbohydrate antigen 724 (CA724) was the most correlative

67 serum tumor biomarker for gastric cancer(Chen et al. 2012). It had been recently reported that

68 serum CA724 was closely correlated to tumor staging of CRC patients(Zhu et al. 2014). But, the

69 relationship between serum CA724 levels and prognosis of CRC remained unclear, especially in

70 the patients with normal CEA levels. Therefore, the aim of the present study was to determine the

71 utility of preoperative serum CA724 levels to predict the prognosis of CRC patients with normal

72 CEA levels.

73 Methods

74 Study population. The medical records of the CRC patients who underwent surgery at the

75 Department of Colorectal Anal Surgery, the First Affiliated Hospital of Guangxi Medical

76 University of between September 2012 and September 2014 were collected. The cases were 
77 chosen according to the following inclusion criteria: 1) histopathological diagnosis of colon or

78 rectal cancer; 2) primary tumor that can be radically removed; 3) complete clinical pathology report and postoperative follow-up data; 4) complete data of preoperative serum oncologic markers. The cases were excluded according to the following criteria: 1) lack of histopathological confirmation of colon or rectal cancer; 2) preoperative neoadjuvant treatment; 3) death cause other than CRC-related; 4) other simultaneous or heterogenic malignant tumors (such as lymphoma, leiomyosarcoma, stromal tumor, melanoma, etc.); 5) no signature on the informed consent form for disposal of biological specimens.

\section{Collection of serum tumor marker and clinicopathological characteristics. Preoperative} fasting venous blood $(4 \mathrm{~mL})$ was collected on the second day after the admission of the patients and centrifuged within $1 \mathrm{~h}$ after collection. Serum that had not be analyzed within $6 \mathrm{~h}$ was stored in a refrigerator at $-20^{\circ} \mathrm{C}$. The serum levels of CEA, CA724, carbohydrate antigen 199 (CA199), carcinoembryonic antigen 125 (CA125), and carcinoembryonic antigen 242 (CA242) were measured using a chemiluminescence immunoassay and the Elecsys 2010 Immunoassay Analyzer (Roche Diagnostics, Risch-Rotkreuz, Switzerland). The following concentrations were considered to indicate high expression: CEA $>5 \mathrm{ng} / \mathrm{mL}, \mathrm{CA} 199>37 \mathrm{U} / \mathrm{mL}, \mathrm{CA} 724>5.7 \mathrm{U} / \mathrm{mL}, \mathrm{CA} 242>20.0$ $\mathrm{U} / \mathrm{mL}$, and $\mathrm{CA} 125>35.0 \mathrm{U} / \mathrm{mL}$. These clinicopathological characteristics were collected in this study including age, gender, pathological tumor stage (pT stage), pathological node stage (pN stage), metastasis, tumor location, venous invasion, perineural invasion, pathologic type, differentiation, postoperative chemoradiotherapy. The patients were staged according to the 
97 American Joint Committee on Cancer staging manual (seventh edition). Radical operation was

98

99

100

101

102

103

104

105

106

107

108

109

110

111

112

113

114

115

116

colorectal resection plus regional lymph node dissection(Cohen 1991; Nascimbeni et al. 2002;

West et al. 2009).

Survival follow-up. Patients were followed-up by telephone or outpatient once every 3 months for the first 2 years after surgery and then every 6 months thereafter. Follow-up examinations included measurements of serum tumor marker levels, chest, abdomen, pelvic imaging by computed tomography, and electronic colonoscopy. The follow-up period was from the time of the discharge to the time of death or July 1,2019 . The censored data of this study was defined as the data of the patients who were survival at the final follow-up deadline. Overall survival (OS) was defined as the time from resection of colorectal cancer to death from colorectal cancer or the censored time. Disease-free survival (DFS) was defined as the time from diagnosis to the first recurrence.

Statistical analyses. All statistical analyses were conducted using IBM SPSS Statistics for Windows, version 24.0 (IBM Corp, Armonk, NY, USA). The Chi-square test was used to identify associations between serum CA724 levels and clinicopathological features. We applied KaplanMeier curve to estimate the survival curve of OS and DFS, which was compared with the log-rank test. Univariate, multivariate, and subgroup survival analysis were performed by using Cox proportional hazards model. All factors that were identified as significant associated with OS and DFS by univariate analysis were subjected to multivariate Cox proportional hazards analysis. Nomograms for predicting OS and DFS were developed using R v3.2.5. A probability $(P)$ value 
117 of $<0.05$ was considered statistically significant. The confidence level of confidence intervals at

118 the $95 \%(95 \% \mathrm{CI})$ was used in this study

119 Ethics statement. The study protocol was approved by the Hospital Ethics Committee of the First

120 Affiliated Hospital of Guangxi Medical University, Guangxi, China-Approval number: 2018(KY-

121 E-086). All patients signed informed consent for collection and analysis of biological specimens.

122 This study was conducted in accordance with the Declaration of Helsinki.

\section{Results}

Clinical characteristics. We collected medical records of $670 \mathrm{CRC}$ patients. The process of case preoperative serum CEA was normal. We excluded patients who had received preoperative adjuvant therapy, incomplete data, non-colorectal cancer deaths and not radical resection. There were 65 patients who were lost to follow-up. Finally, the medical records of 295 CRC patients with normal CEA level were retrospectively reviewed. The median follow-up time of OS was 65 months and DFS was 64 months.

The characteristics of these patients were displayed in Table 1. Among those 295 patients, there were 223 having tumors in the left side and 72 in the right side. The pathological stages of the tumors were as follows: 98 as T1-2, 197 as T3-4, and 186 as N0, 71 as N1, and 38 as N2. The 
136

137

differentiated. Venous invasion or perineural invasion was detected in 48 patients and metastasis was detected in 11 patients.

\section{The correlation between preoperative serum CA724 levels and clinical features. Chi-square} test was used to test the correlation between preoperative serum CA724 levels and clinical features (Table 1), which included age, gender, tumor location, pT stage, pN stage, differentiation, venous invasion, perineural invasion, metastasis and pathologic type. Among these clinicopathological features, only pathological node stage ( $\mathrm{pN}$ stage $)\left(\mathrm{X}^{2}=14.514, P=0.001\right)$ and differentiation $\left(\mathrm{X}^{2}=10.712, P=0.001\right)$ were associated with serum CA724 levels.

Prognostic values of serum tumor markers. In the Kaplan-Meier analysis, the results revealed that the OS and DFS of the patients with high CA724 was poorer than those with normal (Figure 2B, F), but there was no significant difference between CA199, CA242, CA125 high patients and CA199, CA242, CA125 normal patients (Figure 2A, C, D, E, G, H). And there was significant difference between CA724 high patients and those with normal in the Kaplan-Meier analysis of TMN stage III (Figure 3). As shown in Table 2 and Table 3, pT stage, pN stage, metastasis, vascular or neurological invasion, differentiation and serum levels of CA724 were significantly correlated with OS and DFS by univariate analysis. In the multivariate Cox regression analysis of OS, the result indicated that only pT stage $(P=0.036), \mathrm{pN}$ stage $(P<0.001)$, metastasis $(P=0.003)$ and serum CA724 $(P=0.005)$ were independent prognostic factors for CRC patients with normal serum CEA levels. In the multivariate Cox regression analysis of DFS, the result showed the same independent prognostic factors namely pT stage $(P=0.039)$, $\mathrm{pN}$ stage $(P<0.001)$, metastasis 
156

157

158

159

160

161

162

163

164

165

166

167

168

169

170

171

172

173 174

$(P=0.001)$ and serum CA724 $(P=0.001)$ for CRC patients with normal serum CEA levels.

Subgroup analysis of clinical features. The results of multivariate Cox proportional hazards analysis suggested that CA724 was an independent prognostic risk factor for CRC patients with normal CEA. In order to further assess the prognostic value of CA724 in CEA normal patients, CA724 and other factors that identified significant association with DFS and OS by univariate analysis were subjected to multivariate Cox proportional hazards analysis for subgroup analysis of each clinical feature. The statistical results of CA724 in each subgroup were demonstrated in Figure 4 . In the analysis of 11 subgroups, there were statistical significances with DFS in the subgroups of female, age group (>65), T3-T4 stage, no metastasis, right side, no venous invasion and perineural invasion, well/moderate differentiation, no postoperative chemoradiotherapy, while there were statistical significances with OS in the subgroups of gender, age group $(>65)$, T3-T4 stage, N2 stage, tumor location, venous invasion and perineural invasion, protrude type, ulcerative typ, well/moderate differentiation, postoperative chemoradiotherapy.

Nomogram for Predicting Survival Outcomes. Two nomograms were established to evaluate the relationship between CA724 and medical rank in CRC patients with normal CEA (Figure 5). Based on the results of multivariate Cox proportional hazards analysis, only CA724, pT stage, pN stage and metastasis were entered the risk model. The survival rate of 1-5 years could be predicted and CA724 played an important role in the nomogram. 
175

176

177

178

179

180

181

182

183

184

185

186

187

188

189

190

191

192

193

194

\section{Discussion}

More reliable markers were needed to predict the prognosis of CRC patients in a more sufficient manner. The measurement of serum tumor marker levels was a reliable, simple, effective, and economical method to evaluate the prognosis of cancer patients(Huang et al. 2016; Zou \& Qian 2014). CEA, CA199, CA125, CA724, CA242 were commonly used serum biomarkers for preoperative diagnose, postoperative monitoring and efficacy evaluation in gastrointestinal malignancies. But, these serum tumor markers had no specificity for CRC. To the best of our knowledge, there was no a specific serum tumor marker to diagnose CRC despite of the fact that many researchers and scientists had poured great efforts in it. The combined use or supplementary application of the above known serum biomarkers was considered to be potentially effective and practical way to improve the high rate of CRC on diagnosis(Kunizaki et al. 2016; Zhang et al. 2016). CEA was an important serum marker for evaluating prognosis of CRC patients(Lech et al. 2016). However, the high rate of CEA was generally low. According to our retrospective analysis in large series of case investigation, the high rate of preoperative CEA was only $38.04 \%$ in 1969 CRC patients(JiaLiang et al. 2013), which suggested that most of the patients belonged to the preoperative CEA-normal CRC patients and would be lack of efficient serum biomarkers for CRC patients to monitor and follow-up after treatment. In this study, the results of multivariate analyses showed that among several serum tumor markers (CA125, CA199, CA724, and CA242), only CA724 was a statistically significant independent risk factor for the prognosis of CRC patients with normal preoperative serum CEA levels. Generally, the patients with a later TNM stage had a 
195

196

197 198

worse prognosis. In the statistically significant subgroups, the prognosis was poor for CA724-high patients and there was only significant difference between CA724 high patients and those with normal in the TNM stage III. We could hold that CA724 performed an important role in predicting prognosis of CEA-normal patients.

This study was the first to show the predictive value of preoperative serum CA724 levels for the prognosis of CRC patients with normal CEA levels. CA724 was a high molecular weight mucinlike glycoprotein that was upregulated in malignant tumors of the gastrointestinal tract, reproductive system, and lung(Liang et al. 2013). The prognostic value of serum CA724 to detect the recurrence of gastric cancer was first established in the early 1990s(Chen et al. 2014). Han et al(Yanqing et al. 2018) showed that the diagnostic sensitivity was low (0.50) but the specificity was high (0.86) for serum CA724 as a biomarker in the diagnosis of colorectal cancer. It indicated that CA724 still had a close relationship with colorectal cancer. Liu et al(Liu et al. 2015) showed that CA724 was a better predictor of the prognosis of unresectable pancreatic cancer relative to CA199 and CA125, which suggested that the production and release of CA724 might be similar to that in gastrointestinal malignancies.

In addition, serum CA724 levels and $\mathrm{pN}$ stage were independent prognostic factors of CRC, indicating that both were correlated to tumorigenesis. A study by Sun et al(Sun et al. 2017) showed that an elevation in serum CA724 levels suggested that tumor cells had metastasized through the lymphatic or other pathways and had colonized at an another location. The lymph system was a common metastatic pathway of gastrointestinal cancer( $\mathrm{Li}$ et al. 2013). Chen et al(Chen et al. 
215 2016b) reported that serum CA724 might be associated with the overexpression of human

216 epidermal growth factor receptor 2 (HER2). In some malignancies, HER2 was a targeted

217 therapeutic marker. These studies demonstrated that CA724 played an important role in tumor

218 development and might be valuable as a prognostic marker of CRC patients, especially in the CRC

219 patients with normal CEA. This retrospective study was a single cohort study that based on the

220 limited data available. Therefore, it was necessary to expand the sample from self-validation, or

221 further validate our point of view through horizontal multi-center data in the future.

223 Conclusion

224 Preoperative serum CA724 might serve as a potential predictive biomarker for the prognosis of

225 CRC patients with normal CEA levels. And more multicenter studies and statistics were needed to 226 verify.

\section{Abbreviations}

228 CRC, colorectal cancer; CEA, carcinoembryonic antigen; OS, overall survival; CA724, 229 carbohydrate antigen 724; CA199, carbohydrate antigen 199; CA242, carcinoembryonic antigen 230 242; CA125, carcinoembryonic antigen 125. pT stage, pathological tumor stage; pN stage, 231 pathological node stage.

\section{Acknowledgments}


233 The authors thank the Hospital Ethics Committee of the First Affiliated Hospital of Guangxi

234 Medical University for approving this study.

235

236 Disclosure

237 The authors report no conflicts of interest in this work. 
238

239

240

241

242

\section{Reference}

Bray F, Ferlay J, Soerjomataram I, Siegel RL, Torre LA, and Jemal A. 2018. Global cancer statistics 2018: GLOBOCAN estimates of incidence and mortality worldwide for 36 cancers in 185 countries. CA: A Cancer Journal for Clinicians 68:394-424.

Chen W, Zheng R, Baade PD, Zhang S, Zeng H, Bray F, Jemal A, Yu XQ, and He J. 2016a. Cancer statistics in China, 2015. CA: A Cancer Journal for Clinicians 66:115-132.

Benson AB, Venook AP, Al-Hawary MM, Cederquist L, Chen Y-J, Ciombor KK, Cohen S, Cooper HS, Deming D, and Engstrom PF. 2018. NCCN guidelines insights: colon cancer, version 2.2018. Journal of the National Comprehensive Cancer Network 16:359-369.

Feng X, Liu J, Gong Y, Gou K, Yang H, Yuan Y, and Xing C. 2018. DNA repair protein XPA is differentially expressed in colorectal cancer and predicts better prognosis. Cancer medicine 7:2339-2349.

Nakamura Y, and Yoshino T. 2018. Clinical utility of analyzing circulating tumor DNA in patients with metastatic colorectal cancer. The oncologist 23:1310-1318.

Lea D, Håland S, Hagland HR, and Søreide K. 2014. Accuracy of TNM staging in colorectal cancer: a review of current culprits, the modern role of morphology and stepping-stones for improvements in the molecular era. Scandinavian Journal of Gastroenterology 49:1153-1163.

Li J, Guo B-C, Sun L-R, Wang J-W, Fu X-H, Zhang S-Z, Poston G, and Ding K-F. 2014. TNM staging of colorectal cancer should be reconsidered by T stage weighting. World Journal of Gastroenterology: WJG 20:5104.

Jo JC, Ryu MH, Koo DH, Ryoo BY, Kim HJ, Kim TW, Choi KD, Lee GH, Jung HY, and Yook JH. 2013. Serum CA 19 - 9 as a prognostic factor in patients with metastatic gastric cancer. Asia - Pacific Journal of Clinical Oncology 9:324-330.

Kozman MA, Fisher OM, Rebolledo BAJ, Parikh R, Valle SJ, Arrowaili A, Alzahrani N, Liauw W, and Morris DL. 2018. CEA to peritoneal carcinomatosis index $(\mathrm{PCl})$ ratio is prognostic in patients with colorectal cancer peritoneal carcinomatosis undergoing cytoreduction surgery and intraperitoneal chemotherapy: a retrospective cohort study. Journal of Surgical Oncology 117:725-736.

Peng Y, Zhai Z, Li Z, Wang L, and Gu J. 2015. Role of blood tumor markers in predicting metastasis and local recurrence after curative resection of colon cancer. International Journal of Clinical and Experimental Medicine 8:982.

Chen T, Yu L, Li B, Zhang F, Wang Y, Wang X, Liu S, Yang C, Qi H, and Yu M. 2017. Elevated Preoperative Carcinoembryonic Antigen and Vascular Endothelial Growth Factor Predict Shorter Survival in Patients with 
Sigmoid Colon Carcinoma. Clinical laboratory 63:445-451.

Shinkins B, Nicholson BD, Primrose J, Perera R, James T, Pugh S, and Mant D. 2017. The diagnostic accuracy of a single CEA blood test in detecting colorectal cancer recurrence: Results from the FACS trial. PloS One 12:e0171810.

Huang S-C, Huang S-F, Chen Y-T, Chang Y, Chiu Y-T, Chang I-C, Wu H-DI, and Chen J-S. 2017. Overexpression of MutL homolog 1 and MutS homolog 2 proteins have reversed prognostic implications for stage I-II colon cancer patients. Biomedical journal 40:39-48.

Huang E-Y, Chang J-C, Chen H-H, Hsu C-Y, Hsu H-C, and Wu K-L. 2018. Carcinoembryonic antigen as a marker of radioresistance in colorectal cancer: a potential role of macrophages. BMC Cancer 18:321.

Chen X-Z, Zhang W-K, Yang K, Wang L-L, Liu J, Wang L, Hu J-K, Zhang B, Chen Z-X, and Chen J-P. 2012. Correlation between serum CA724 and gastric cancer: multiple analyses based on Chinese population. Molecular Biology Reports 39:9031-9039.

Zhu Z, Chen Z, Chen C, Yang Z, Xuan W, Hou Y, Zuo Y, and Ren S. 2014. Opposite variation tendencies of serum CA724 levels in patients with colon and rectal carcinoma. Molecular and clinical oncology 2:139-145.

Cohen A. 1991. Surgical considerations in patients with cancer of the colon and rectum. Seminars in Oncology. p 381.

West NP, Hohenberger W, Weber K, Perrakis A, Finan PJ, and Quirke P. 2009. Complete mesocolic excision with central vascular ligation produces an oncologically superior specimen compared with standard surgery for carcinoma of the colon. Journal of Clinical Oncology 28:272-278.

Nascimbeni R, Burgart L, Nivatvongs S, and Larson DR. 2002. Risk of lymph node metastasis in T1 carcinoma of the colon and rectum. Diseases of the Colon and Rectum 45:200-206.

Zou L, and Qian J. 2014. Decline of serum CA724 as a probable predictive factor for tumor response during chemotherapy of advanced gastric carcinoma. Chinese Journal of Cancer Research 26:404.

Huang C-J, Jiang J-K, Chang S-C, Lin J-K, and Yang S-H. 2016. Serum CA125 concentration as a predictor of peritoneal dissemination of colorectal cancer in men and women. Medicine 95.

Zhang B, Liang X, Gao H, Ye L, and Wang Y. 2016. Models of logistic regression analysis, support vector machine, and back-propagation neural network based on serum tumor markers in colorectal cancer diagnosis. Genet $\mathrm{Mol}$ Res 15.

Kunizaki M, Sawai T, Takeshita H, Tominaga T, Hidaka S, To K, Miyazaki T, Hamamoto R, Nanashima A, and Nagayasu T. 2016. Clinical value of serum p53 antibody in the diagnosis and prognosis of colorectal cancer. Anticancer 
Research 36:4171-4175.

297

298

299

300

301

302

303

304

305

306

307

308

309

310

311

312

313

314

315

316

317

318

319

320

Lech G, Słotwiński R, Słodkowski M, and Krasnodębski IW. 2016. Colorectal cancer tumour markers and biomarkers: Recent therapeutic advances. World Journal of Gastroenterology 22:1745.

JiaLiang G, Feng G, YunFei C, Heng L, and YongSheng G. 2013. Clinicopathologic and epidemiologic analysis of 1220 cases of the colorectal carcinoma with negative preoperative carcinoembryonic antigen. Modern Preventive Medicine 40:1772-1774.

Liang Y, He M, Fan X, Ye W, Yang Z, and Zhong R. 2013. An abnormal elevation of serum CA72-4 by ganoderma lucidum spore powder. Annals of Clinical and Laboratory Science 43:337-340.

Chen X-Z, Zhang W-H, Yang K, Zhang B, Chen Z-X, Chen J-P, Zhou Z-G, and Hu J-K. 2014. Quantitative comparisons of summary receiver operating characteristics (sROC) curves among conventional serological tumor biomarkers for predicting gastric cancer in Chinese population. Tumor Biology 35:9015-9022.

Yanqing H, Cheng D, and Ling X. 2018. Serum CA72-4 as a biomarker in the diagnosis of colorectal cancer: A metaanalysis. Open Medicine 13:164-171.

Liu P, Zhu Y, and Liu L. 2015. CA724 is a novel factor for predicting the unresectability in pancreatic adenocarcinoma. International Journal of Clinical and Experimental Pathology 8:15112.

Sun Z-Q, Ma S, Zhou Q-B, Yang S-X, Chang Y, Zeng X-Y, Ren W-G, Han F-H, Xie X, and Zeng F-Y. 2017. Prognostic value of lymph node metastasis in patients with T1-stage colorectal cancer from multiple centers in China. World Journal of Gastroenterology 23:8582.

Li F, Li S, Wei L, Liang X, Zhang H, and Liu J. 2013. The correlation between pre-operative serum tumor markers and lymph node metastasis in gastric cancer patients undergoing curative treatment. Biomarkers: Biochemical Indicators of Exposure, Response, and Susceptibility to Chemicals 18:632-637.

Chen X-Z, Zhang W-H, Chen H-N, Liu J-P, He D, Liu Y, Liu K, Chen X-L, Mo X-M, and Zhou Z-G. 2016b. Associations between serum CA724 and HER2 overexpression among stage II-III resectable gastric cancer patients: an observational study. Oncotarget 7:23647. 


\section{Table 1 (on next page)}

Comparison of baseline clinicopathological characteristics based on CA724 in CEAnormal CRC patients.

Comparison of baseline clinicopathological characteristics based on CA724 in CEA-normal CRC patients. 


\begin{tabular}{|c|c|c|c|c|c|}
\hline \multirow{2}{*}{ Feature } & \multirow{2}{*}{ case } & \multicolumn{2}{|c|}{ CA724 } & \multirow{2}{*}{$\chi^{2}$} & \multirow{2}{*}{$\mathrm{p}$} \\
\hline & & Normal (242) & High (53) & & \\
\hline Gender & & & & 0.026 & 0.872 \\
\hline Male & $181(61.36 \%)$ & $149(61.6 \%)$ & $32(60.4 \%)$ & & \\
\hline Female & $114(38.64 \%)$ & $93(38.4 \%)$ & $21(39.6 \%)$ & & \\
\hline Age (Year) & & & & 3.424 & 0.064 \\
\hline$\leq 65$ & $161(54.58 \%)$ & $126(52.1 \%)$ & $35(66.0 \%)$ & & \\
\hline$>65$ & $134(45.42 \%)$ & $116(47.9 \%)$ & $18(34.0 \%)$ & & \\
\hline pT stage & & & & 0.594 & 0.441 \\
\hline $\mathrm{T} 1-2$ & $98(33.22 \%)$ & $78(32.2 \%)$ & $20(37.7 \%)$ & & \\
\hline T3-4 & $197(66.78 \%)$ & $164(67.8 \%)$ & $33(62.3 \%)$ & & \\
\hline pN stage & & & & 14.514 & 0.001 \\
\hline No & $186(63.05 \%)$ & $156(64.5 \%)$ & $30(56.6 \%)$ & & \\
\hline N1 & $71(24.07 \%)$ & $63(26.0 \%)$ & $8(15.1 \%)$ & & \\
\hline $\mathrm{N} 2$ & $38(12.88 \%)$ & $23(9.5 \%)$ & $15(28.3 \%)$ & & \\
\hline Metastasis & & & & 0.611 & 0.435 \\
\hline No & $284(96.27 \%)$ & $232(95.9 \%)$ & $52(98.1 \%)$ & & \\
\hline Yes & $11(3.73 \%)$ & $10(4.1 \%)$ & $1(1.9 \%)$ & & \\
\hline Tumor location & & & & 0.001 & 0.982 \\
\hline Left side & $223(75.6 \%)$ & $183(75.6 \%)$ & $40(75.5 \%)$ & & \\
\hline Right side & $72(24.4 \%)$ & $59(24.4 \%)$ & $13(24.5 \%)$ & & \\
\hline Perineural/Vascular invasion & & & & 3.233 & 0.072 \\
\hline
\end{tabular}




$\begin{array}{llll}\text { No } & 247(83.73 \%) & 207(85.5 \%) & 40(75.5 \%) \\ \text { Yes } & 48(16.27 \%) & 35(14.5 \%) & 13(24.5 \%)\end{array}$

\begin{tabular}{|c|c|c|c|c|c|}
\hline Pathological type & & & & 2.101 & 0.350 \\
\hline Protrude type & $48(16.27 \%)$ & $43(17.8 \%)$ & $14(26.4 \%)$ & & \\
\hline Infiltrating type & $48(16.27 \%)$ & $29(12.0 \%)$ & $6(11.3 \%)$ & & \\
\hline Ulcerative type & $203(68.82 \%)$ & $170(70.2 \%)$ & $33(62.3 \%)$ & & \\
\hline Differentiation & & & & 10.712 & 0.001 \\
\hline Poor & $34(11.53 \%)$ & $21(8.7 \%)$ & $13(24.5 \%)$ & & \\
\hline Medium/High & $261(88.47 \%)$ & $221(91.3 \%)$ & $40(75.5 \%)$ & & \\
\hline
\end{tabular}

Abbreviations: CEA: carcinoembryonic antigen; CRC: colorectal cancer; pT stage: pathological tumor stage; $\mathrm{pN}$ stage: pathological node stage 


\section{Table 2 (on next page)}

Univariate and multivariate overall survival analyses of clinicopathological covariates in CRC patients.

Univariate and multivariate overall survival analyses of clinicopathological covariates in CRC patients. 


\begin{tabular}{|c|c|c|c|c|}
\hline \multirow{3}{*}{ Feature } & \multicolumn{4}{|c|}{ Overalll Survival } \\
\hline & \multicolumn{2}{|c|}{ Univariate analysis } & \multicolumn{2}{|c|}{ Multivariate analysis } \\
\hline & HR $(95 \% \mathrm{CI})$ & $p$ & $\mathrm{HR}(95 \% \mathrm{CI})$ & $p$ \\
\hline Gender & & 0.885 & & \\
\hline Male & 1 & & & \\
\hline Female & $0.970(0.637-1.475)$ & & & \\
\hline Age (Year) & & 0.161 & & \\
\hline$\leq 65$ & 1 & & & \\
\hline$>65$ & $1.338(0.891-2.011)$ & & & \\
\hline pT stage & & 0.002 & & 0.039 \\
\hline $\mathrm{T} 1-2$ & 1 & & 1 & \\
\hline T3-4 & $2.230(1.347-3.693)$ & & $1.761(1.029-3.014)$ & \\
\hline pN stage & & $<0.001$ & & $<0.001$ \\
\hline N0 & 1 & & 1 & \\
\hline $\mathrm{N} 1$ & $1.788(1.084-2.949)$ & & $1.589(0.943-2.676)$ & \\
\hline $\mathrm{N} 2$ & $5.524(3.391-8.998)$ & & $3.995(2.304-6.927)$ & \\
\hline
\end{tabular}




\begin{tabular}{|c|c|c|c|c|}
\hline Metastasis & & $<0.001$ & & 0.001 \\
\hline No & 1 & & 1 & \\
\hline Yes & $4.078(1.964-8.467)$ & & $3.509(1.624-7.582)$ & \\
\hline Tumor location & & 0.419 & & \\
\hline Left side & 1 & & & \\
\hline Right side & $1.208(0.764-1.911)$ & & & \\
\hline $\begin{array}{l}\text { Perineural/Vascular } \\
\text { invasion }\end{array}$ & & 0.005 & & 0.179 \\
\hline No & 1 & & 1 & \\
\hline Yes & $1.960(1.223-3.141)$ & & $1.418(0.852-2.358)$ & \\
\hline Pathological type & & 0.697 & & \\
\hline Protrude type & 1 & & & \\
\hline Infiltrating type & $1.354(0.634-2.894)$ & & & \\
\hline Ulcerative type & $1.234(0.705-2.162)$ & & & \\
\hline Differentiation & & 0.019 & & 0.257 \\
\hline Poor & 1 & & 1 & \\
\hline
\end{tabular}




\begin{tabular}{|c|c|c|c|c|}
\hline Medium/High & $0.523(0.305-0.898)$ & & $1.427(0.772-2.639)$ & \\
\hline $\begin{array}{c}\text { Postoperative } \\
\text { chemoradiotherapy }\end{array}$ & & 0.786 & & \\
\hline No & 1 & & & \\
\hline Yes & $1.058(0.704-1.591)$ & & & \\
\hline CA199 & & 0.060 & & \\
\hline Normal & 1 & & & \\
\hline High & $1.790(0.976-3.283)$ & & & \\
\hline CA724 & & $<0.001$ & & 0.001 \\
\hline Normal & 1 & & 1 & \\
\hline High & $2.271(1.450-3.557)$ & & $2.261(1.379-3.708)$ & \\
\hline $\mathrm{CA} 242$ & & 0.149 & & \\
\hline Normal & 1 & & & \\
\hline High & $1.520(0.861-2.685)$ & & & \\
\hline CA125 & & 0.058 & & \\
\hline Normal & 1 & & & \\
\hline
\end{tabular}


High $\quad 1.945(0.977-3.868)$

1 Abbreviations: CEA: carcinoembryonic antigen; CRC: colorectal cancer; pT stage: pathological tumor stage; pN stage: pathological node stage. 


\section{Table 3(on next page)}

Univariate and multivariate disease-free survival analyses of clinicopathological covariates in CRC patients.

Univariate and multivariate disease-free survival analyses of clinicopathological covariates in CRC patients. 


\begin{tabular}{|c|c|c|c|c|}
\hline \multirow{3}{*}{ Feature } & \multicolumn{4}{|c|}{ Disease-free survival } \\
\hline & \multicolumn{2}{|c|}{ Univariate analysis } & \multicolumn{2}{|c|}{ Multivariate analysis } \\
\hline & HR $(95 \% \mathrm{CI})$ & $p$ & HR $(95 \% \mathrm{CI})$ & $p$ \\
\hline Gender & & 0.898 & & \\
\hline Male & 1 & & & \\
\hline Female & $0.974(0.645-1.469)$ & & & \\
\hline Age (Year) & & 0.403 & & \\
\hline$\leq 65$ & 1 & & & \\
\hline$>65$ & $1.186(0.795-1.770)$ & & & \\
\hline pT stage & & 0.001 & & 0.036 \\
\hline $\mathrm{T} 1-2$ & 1 & & 1 & \\
\hline T3-4 & $2.244(1.371-3.675)$ & & $1.751(1.038-2.956)$ & \\
\hline pN stage & & $<0.001$ & & $<0.001$ \\
\hline No & 1 & & 1 & \\
\hline N1 & $1.944(1.195-3.161)$ & & $1.714(1.031-2.849)$ & \\
\hline $\mathrm{N} 2$ & $5.462(3.361-8.874)$ & & $3.820(2.192-6.660)$ & \\
\hline
\end{tabular}




\begin{tabular}{|c|c|c|c|c|}
\hline Metastasis & & $<0.001$ & & 0.003 \\
\hline No & 1 & & 1 & \\
\hline Yes & $4.629(2.231-9.604)$ & & $3.292(1.514-7.154)$ & \\
\hline Tumor location & & 0.245 & & \\
\hline Left side & 1 & & & \\
\hline Right side & $1.302(0.835-2.033)$ & & & \\
\hline $\begin{array}{c}\text { Perineural/Vascular } \\
\text { invasion }\end{array}$ & & 0.009 & & 0.285 \\
\hline No & 1 & & 1 & \\
\hline Yes & $1.874(1.172-2.996)$ & & $1.316(0.795-2.178)$ & \\
\hline Pathological type & & 0.793 & & \\
\hline Protrude type & 1 & & & \\
\hline Infiltrating type & $1.256(0.594-2.655)$ & & & \\
\hline Ulcerative type & $1.187(0.688-2.046)$ & & & \\
\hline Differentiation & & 0.023 & & 0.294 \\
\hline Poor & 1 & & 1 & \\
\hline
\end{tabular}




\begin{tabular}{|c|c|c|c|c|}
\hline Medium/High & $0.536(0.313-0.918)$ & & $1.383(0.754-2.536)$ & \\
\hline $\begin{array}{c}\text { Postoperative } \\
\text { chemoradiotherapy }\end{array}$ & & 0.706 & & \\
\hline No & 1 & & & \\
\hline Yes & $1.080(0.723-1.614)$ & & & \\
\hline CA199 & & 0.069 & & \\
\hline Normal & 1 & & & \\
\hline High & $1.753(0.957-3.210)$ & & & \\
\hline CA724 & & 0.001 & & 0.005 \\
\hline Normal & 1 & & 1 & \\
\hline High & $2.097(1.342-3.275)$ & & $2.028(1.238-3.320)$ & \\
\hline CA242 & & 0.070 & & \\
\hline Normal & 1 & & & \\
\hline High & $1.664(0.959-2.889)$ & & & \\
\hline CA125 & & 0.066 & & \\
\hline Normal & 1 & & & \\
\hline
\end{tabular}


High

$1.906(0.959-3.787)$

1 Abbreviations: CEA: carcinoembryonic antigen; CRC: colorectal cancer; pT stage: pathological tumor stage; pN stage: pathological node stage. 
Figure 1

The process of case inclusion and exclusion in this study.

The process of case inclusion and exclusion in this study.

670 Patients with CRC patients between September 2012 and September 2014

276 patients with high preoperative CEA

394 patients with normal preoperative serum CEA

8 patients received preoperative neoadjuvant treatment

4 patients died of non-CRC

11 patients had incomplete data

65 patients were lost to follow-up

11 patients were not radical resection

295 CRC patients with normal CEA level were retrospectively reviewed 
Figure 2

Comparison of OS rate and DFS rate of CRC patients with normal CEA levels according to CA724, CA199, CA242, CA125.

The results of the Kaplan-Meier analysis revealed that the OS rate and DFS rate of the patients with high CA724 was poorer than those with normal, but there was no significant difference between CA199, CA242, CA125 high patients and CA199, CA242, CA125 normal patients $(P<0.05)$.
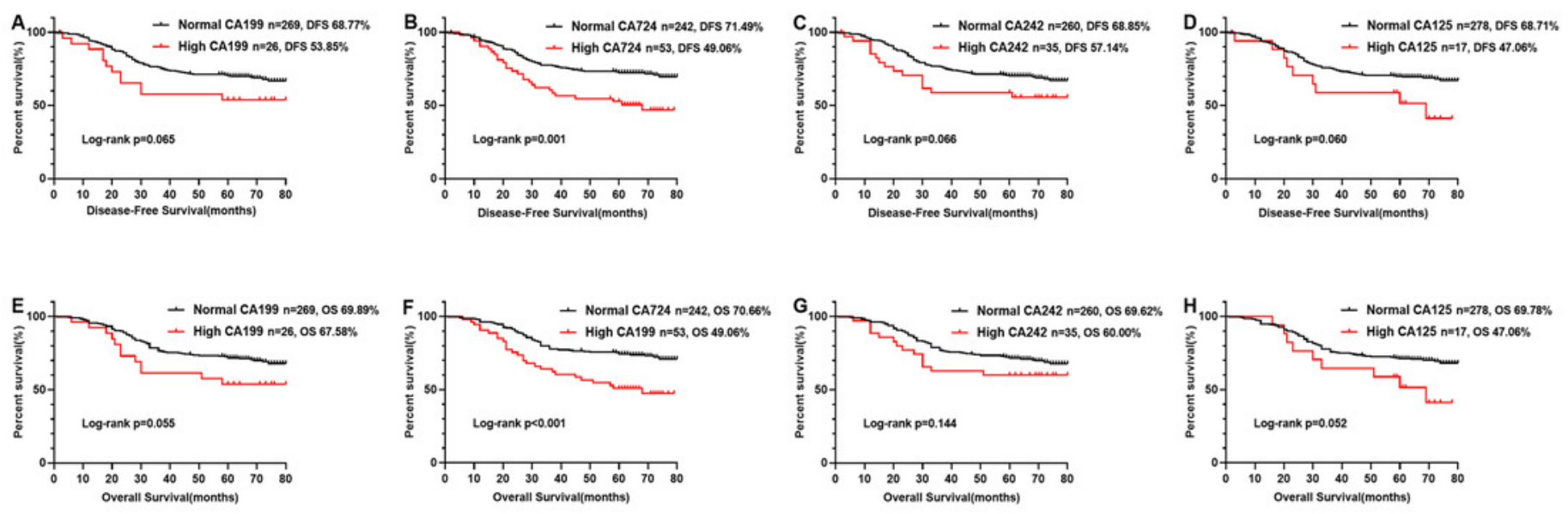
Figure 3

Comparison of OS and DFS of TMN stage according to CA724.

There was significant difference between CA724 high patients and those with normal in the Kaplan-Meier analysis of TMN stage III $(P<0.05)$.

Figure 4

The statistical results of CA724 in each subgroup.

In the analysis of 11 subgroups, there were statistical significances with DFS (A) in the subgroups of female, age group (>65), T3-T4 stage, no metastasis, right side, no venous invasion and perineural invasion, well/moderate differentiation, no postoperative chemoradiotherapy. There were statistical significances with OS (B) in the subgroups of gender, age group (>65), T3-T4 stage, N2 stage, tumor location, venous invasion and perineural invasion, protrude type, ulcerative typ, well/moderate differentiation, postoperative chemoradiotherapy.
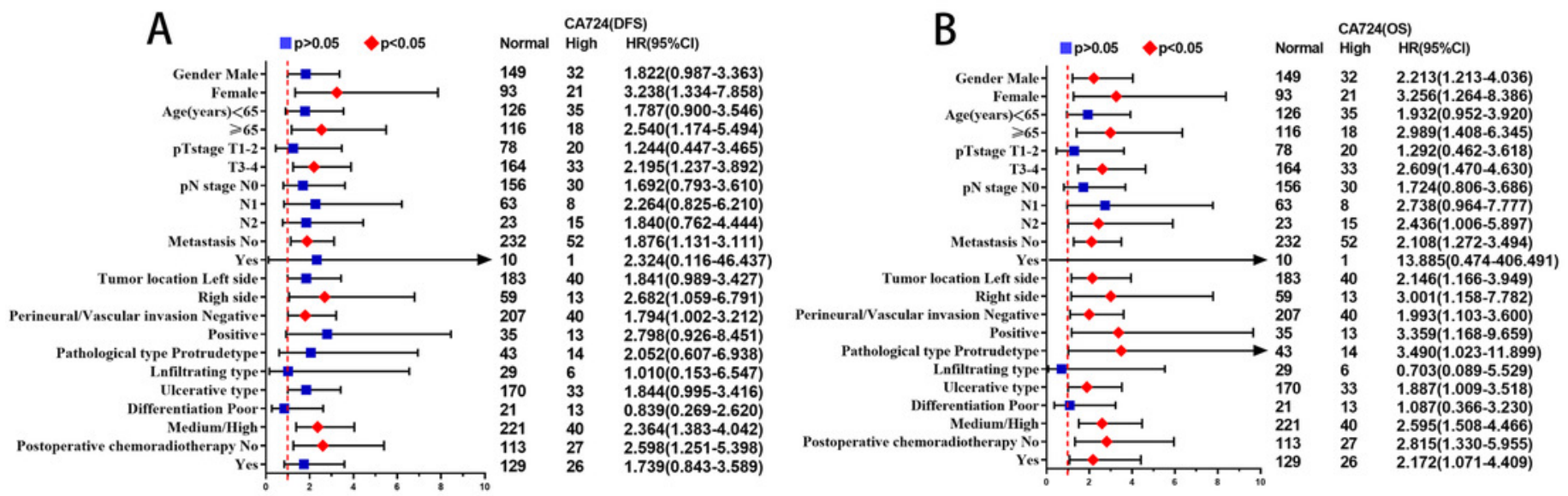
Figure 5

The Nomogram for Predicting Survival Outcomes.

Two nomograms were established to evaluate the relationship between CA724 and medical rank in CRC patients with normal CEA. The survival rate of 1-5 years disease-free survival (A) and 1-5 years overall survival (B) could be predicted.

A

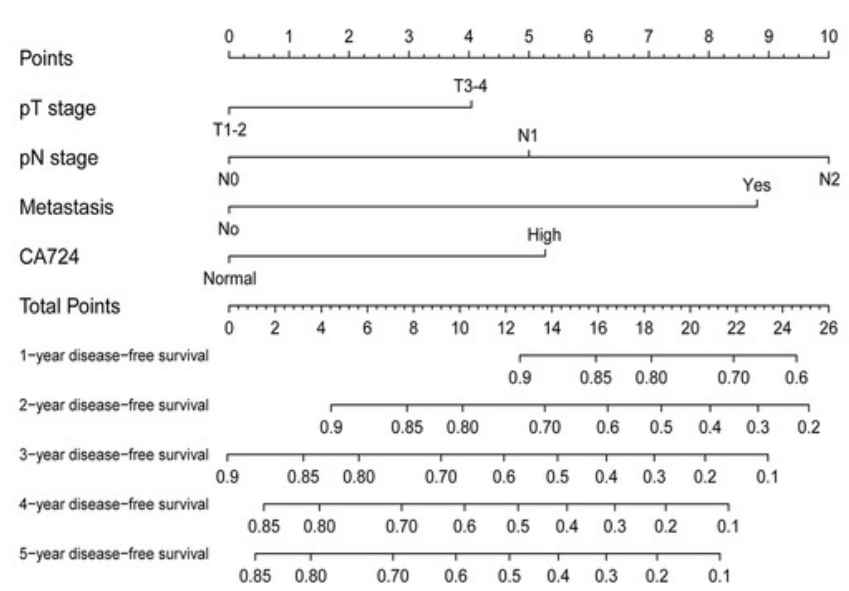

B

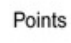

pT stage

$\mathrm{pN}$ stage

Metastasis

CA724

Total Points

1-year overall survival

2-year overall survival

3-year overall survival

4-year overall survival

5-year overall survival

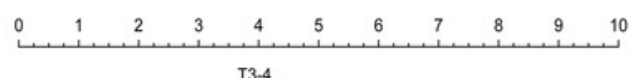

T3-4



High

orma

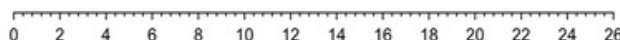

$0.9 \quad 0.85 \quad 0.80 \quad 0.70$

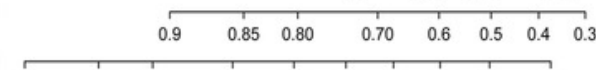

$\begin{array}{lllllllll}0.85 & 0.80 & 0.70 & 0.6 & 0.5 & 0.4 & 0.3 & 0.2 & 0.1\end{array}$ 